ing more widely the nature of neural influences during the development of synapses.

Synapses newly formed in culture between spatially separated explants of chick muscle and spinal cord were first demonstrated, morphologically, by James and Tresman in 1968 (Nature, 220, 384). Cultures of chick embryonic muscle, previously dissociated into individual cells by mild trypsin digestion, have since been shown by several workers to form neuromuscular synapses capable of transmitting impulses when grown with fragments of spinal cord or similarly separated spinal neurones. At mature neuromuscular junctions, transmission normally occurs by the release from the nerve terminal, in response to the arrival of nerve impulses, of discrete quantities of a chemical mediator, acetylcholine (ACh), which acts with receptors at the specialized "end-plate" sites on the muscle membrane causing a fall in the membrane potential (called the "endplate potential" or e.p.p.), which triggers activity in the muscle. Release of ACh at a low level occurs continuously and gives rise to small fluctuations in the muscle membrane potential, known as miniature end-plate potentials, or m.e.p.p.s, but the rate of release of $\mathrm{ACh}$ packets is vastly increased by activity in the nerve terminals, such that the m.e.p.p.s summate to produce potential changes large enough to excite the muscle.

Microelectrode insertions into cultured chick muscle fragments or myoblasts have revealed both m.e.p.p.s, occurring spontaneously, and e.p.p.s, in response to electrical stimulation of spinal cord fragments or nerve fibres, soon after the formation of nervemuscle contacts, and in some cases even before the muscles themselves were capable of contracting (Robbins and Yonezawa, Science, 172, 395; 1971). These potentials could be blocked by $d$-tubocurarine, a well-known antagonist to $\mathrm{ACh}$ at the receptors of the mature neuromuscular junction. This suggests that the potentials were caused by release of $\mathrm{ACh}$ by a process similar to that occurring in vivo during normal neuromuscular transmission.

Adult skeletal muscle fibres are normally sensitive to ACh only at the small end-plate regions where contact is established with nerve endings. But if a muscle is deprived of its nerve supply it becomes responsive to $\mathrm{ACh}$ along the entire length of its surface. Conversely, if the motor nerve fibres are allowed to regenerate into a denervated muscle, the sensitive area shrinks progressively and becomes restricted to the junctional region. Similarly, foetal muscle fibres are uniformly sensitive to $\mathrm{ACh}$, but after birth there is a retraction of sensitivity, this occurring in parallel with the development of the characteristic end-plate structure.

In the experiments of Kano and Shimada, the ACh sensitivity of muscle fibres grown in culture from initially dissociated myoblasts was tested by iontophoretic application together with intracellular recording. It was found that, like foetal and denervated muscle, cultured fibres grown in the absence of neural tissue gave responses to $\mathrm{ACh}$ along their entire length, but fibres grown in the presence of spinal cord fragments, and which showed morphological evidence of nerve-muscle contacts, were sensitive to $\mathrm{ACh}$ only at those regions of contact.

This suggests that neuromuscular connexions developed in vitro are similar to those of the adult animal not only in respect of transmission of impulses, but also in respect of restriction of chemosensitivity.

The nature of the influence causing this selection process is still controversial. Contact with nerve endings seems to be essential, but this could provide a number of possible factors. It was originally suggested that there might be release of some chemical from the nerve endings, loosely called a "trophic substance", but to date no such substance has been successfully identified. It is possible that ACh itself may be the elusive factor, but recent experiments have suggested that it might be impulse activity in nerve endings, and hence in muscles, which is critical in restricting sensitivity, because, in animals in which conduction of impulses in particular motor nerve terminals was blocked with a local anaesthetic, but in which the corresponding neuromuscular junctions remained structurally and functionally intact, the entire surface of the muscle fibres involved became sensitive to applied ACh (L $\phi$ mo and Rosenthal, $J$. Physiol., 216, 52P; 1971).

\title{
Genetics of Immunological Responsiveness
}

THE capacity to make an immune response is affected by several basic variables such as the nature of the responding organism, and the quantity, quality and route of introduction of the antigen. Aside from the phylogenetic studies, which have revealed variation between species in the capacity to respond to particular antigens, great interest has developed in recent years in the discontinuous variation in immunological responsiveness which can be revealed within species. Genetic studies of responsiveness have appeared and there has been much talk of "responders" and "non-responders". One hope from these studies is that more information will accrue about the nature of the immune response by elucidation of its mode of genetic determination. The genetic basis of susceptibility to disease may also be clarified. Gasser and Schreffler, in next Wednesday's Nature New Biology (February 2), present a study on the effect that the $H-2$ locus can have in mice on the capacity to respond to Ea-1 antigens.

In his earlier studies, Gasser has shown that CBA and BALB/c mice are incapable of producing an immune response to the Ea-1 antigens which are present on the erythrocytes of certain wild mice but which are not, as far as can be determined, found in either $\mathrm{BALB} / \mathrm{c}$ or $\mathrm{CBA}$ mice. In contrast, most YBR mice do respond. Responsiveness for an individual mouse seems to be an all-or-nothing response. All the strains involved in the test are inbred and therefore presumed to be genetically homogeneous. Crosses of YBR and CBA mice revealed that responsiveness was a recessive characteristic which further studies showed was controlled by a single Mendelian gene designated $I r-2$. The failure of some YBR mice to respond was not adequately explained but was thought to be related to non-genetic factors. Gasser went on to show that the responder trait behaved as a dominant characteristic in crosses between YBR and $\mathrm{C} 57 \mathrm{Bl} / 10$ (B10) mice. But further crosses between the $F 1$ animals and backcrosses to the parental generations indicated that a single gene hypothesis could not account for the results obtained. Two-way selection experiments (breeding from responders or nonresponders) successfully produced high and low responding lines establishing without doubt that genetic factors were, however, involved.

In their latest article Gasser and Schreffler have continued by typing the animals in the $F 2$ generation of the cross between YBR and B10 for their $\mathrm{H}-2$ histocompatibility antigens. There was found a significant association between the responsive trait and those animals which carried the $H-2^{d}$ allele. Gasser and Schreffler then repeated the cross between YBR and B10 but used this time a congenic variant B10 D2 which has the $H-2^{d}$ allele rather than $H-2^{b}$. The results were clear-cut and indicated that the $I r-2$ gene segregated in the expected manner when the $H-2^{d}$ gene was homozyous throughout (the YBR strain is itself $H-2^{d}$ ).

These results, aside from the interest of the finding that the $H$-2 locus can have an effect on immunological responsiveness, also hint at the possibility that apparently complex modes of inheritance of responder traits may on analysis prove to have single gene bases, the expression of which is susceptible to modification by the products of other gene loci. 